\title{
DYNAMICAL TRAJECTORIES AND CURVATURE TRAJECTORIES*
}

\author{
BY EDWARD KASNER
}

1. Introduction. In this paper we compare two important types of triply infinite families of plane curves, dynamical families and curvature families. Both types are projectively invariant, so that our subject belongs to the projective differential geometry of systems of curves.

A family of dynamical trajectories consists of the $\infty^{3}$ possible paths of a particle moving in a general field of force, initial position and velocity being arbitrary. If $\phi(x, y)$ and $\psi(x, y)$ are the components of force, the equations of motion are

$$
\ddot{x}=\phi(x, y), \quad \ddot{y}=\psi(x, y) .
$$

The differential equation of the trajectories $\dagger$ is found by eliminating the time from $\left(1^{\prime}\right)$ :

$$
\text { (1) }\left(\psi-y^{\prime} \phi\right) y^{\prime \prime \prime}=\left\{\psi_{x}+\left(\psi_{y}-\phi_{x}\right) y^{\prime}-\phi_{y} y^{\prime 2}\right\} y^{\prime \prime}-3 \phi y^{\prime \prime 2} \text {. }
$$

To define curvature trajectories we start with an arbitrary doubly infinite family of curves, that is, a general differential equation of second order:

$$
y^{\prime \prime}=F\left(x, y, y^{\prime}\right) .
$$

A curvature trajectory of this family is a curve which is drawn so as to have at each point $c$ times the curvature of the member of the family to which it is tangent at that point, $c$ remaining constant along the trajectory. For a given value of $c$ there will be a set of $\infty^{2}$ trajectories, (one in each direction through each point). By varying $c$ we obtain $\infty^{1}$ such sets. Hence a given doubly infinite family $\left(2^{\prime}\right)$ generates a triply infinite family of curvature trajectories.

* Presented to the Society, April 26, 1919, under the title $A$ characteristic property of central forces. See abstract, this Bulletin, vol. 25 (1919), p. 443.

$\dagger \mathrm{E}$. Kasner, The trajectories of dynamics, Transactions of this Society, vol. 7 (1906), pp. 401-424. Also Differential-Geometric Aspects of Dynamics, Princeton Colloquium Lectures on Mathematics, 1913, especially Chapters 1 and 3, where some of the properties are stated in projective language. A new edition, published by this Society, has just appeared. 
The $\infty^{2}$ trajectories that can be drawn for a fixed value of the curvature ratio $c$ satisfy the equation $y^{\prime \prime}=c F\left(x, y, y^{\prime}\right)$, (since curvature at a given direction element is proportional to $\left.y^{\prime \prime}\right)$. If we differentiate this and eliminate the parameter $c$, we have the differential equation of all the curvature trajectories of $\left(2^{\prime}\right)$ :

$$
F y^{\prime \prime \prime}=\left(F_{x}+F_{y} y^{\prime}\right) y^{\prime \prime}+F_{y^{\prime}} y^{\prime \prime 2} .
$$

Thus a two-parameter family of curves gives rise by a geometrical construction to $\infty^{3}$ curvature trajectories, just as for instance a one-parameter family gives rise to $\infty^{2}$ isogonal trajectories.* As a simple example, the original family $\left(2^{\prime}\right)$ might be the $\infty^{2}$ unit circles. Then it is easy to see from the definition that their curvature trajectories would be all the $\infty^{3}$ circles of the plane.

We have discussed the projective character of dynamical families in the references. The concept of curvature families involves also a projective concept. This is an immediate consequence of Mehmke's theorem, which states that if two curves are tangent at a point, the ratio of curvatures is a projective invariant. In fact this theorem implies that the entire process of construction of curvature trajectories has projective meaning.

Now we observe that (1) and (2) are both of the general form

$$
y^{\prime \prime \prime}=G\left(x, y, y^{\prime}\right) y^{\prime \prime}+H\left(x, y, y^{\prime}\right) y^{\prime \prime 2},
$$

but with different functions $G$ and $H$. This resemblance between the equations suggests the problem of determining all triply infinite families of curves which are at once dynamical trajectories and curvature trajectories. $\dagger$

The answer is interesting and physically significant. The common families are exactly the trajectories of all central fields of force. For the curvature families this means that $\left(2^{\prime}\right)$ takes one of the special forms $\left(12_{1}\right)$ or $\left(12_{2}\right)$ below.

* The special case of orthogonal trajectories has an analog in curvaturereversing trajectories (curvature ratio $c=-1$, or $Y^{\prime \prime}=-y^{\prime \prime}$ ). In either case the square of the operation of taking trajectories is the identity, so the relation is involutorial.

$\dagger$ We assume that the field in $\left(1^{\prime}\right)$ is not null, and that $F$ in $\left(2^{\prime}\right)$ is not identically zero; these degenerate cases give merely the $\infty^{2}$ straight lines and so correspond to each other. 
The result amounts to a new geometrical characterization of the trajectories of central fields. They are those families of dynamical trajectories whose $\infty^{3}$ curves can be analyzed into a series of sets, each set containing $\infty^{2}$ curves, in such a way that one of the sets will generate the others by the simple process of multiplication of curvatures described above.

We observe that this analysis of the trajectories of a central field also has physical meaning. It can be shown to be identical with the grouping based on the value of the area-sweeping constant, (or on the constant of angular momentum).

2. Geometric Properties of Dynamical Families. For the proof, we employ the following sets of geometric properties, which characterize dynamical families among all triply infinite families of curves (see references).

I. If to each of the $\infty^{1}$ curves having a given lineal element in common the osculating parabola is drawn at that element, the foci will lie on a circle through the point of the element.

II. There exists for each point $(x, y)$ of the plane a certain direction $\omega$ (that of the force) such that the angle between this direction and the tangent to the focal circle corresponding to any element $\left(x, y, y^{\prime}\right)$ at the given point is bisected by that element.

III. The locus of the centers of the $\infty^{1}$ circles corresponding to the lineal elements at a given point is a conic with that point as a focus.

IV. In each direction through a given point $O$ there passes one trajectory which has contact of third order with its circle of curvature. The locus of the centers of the $\infty^{1}$ hyperosculating circles, obtained by varying the initial direction, is a conic passing through the given point in the direction of the force. (But III and IV are equivalent.)

$\mathrm{V}$. Of the curves which pass through a given point in the direction of the force at that point, there is one which has contact of the third order with its circle of curvature; the radius of curvature of this curve is three times the radius of curvature of the line of force (that is, integral curve of the direction assigned to each point by II) passing through the given point.

VI. When the point $O$ is moved, the associated conic described in property IV changes in the following manner. Take any two fixed perpendicular directions for the $x$ direction and 
the $y$ direction; through $O$ draw lines in these directions meeting the conic again at $A$ and $B$, respectively. Also construct the normal at $O$ meeting the conic again at $N$. At $A$ draw a line in the $y$ direction meeting this normal in some point $A^{\prime}$, and at $B$ draw a line in the $x$ direction meeting the normal in some point $B^{\prime}$. The variation property referred to takes the form

$$
\frac{\partial}{\partial x} \frac{1}{A A^{\prime}}+\frac{\partial}{\partial y} \frac{1}{B B^{\prime}}+\frac{\omega \omega_{x y}-\omega_{x} \omega_{y}}{3 \omega^{2}}=0,
$$

where $A A^{\prime}$ and $B B^{\prime}$ denote distances between points, and where $\omega$ denotes the slope of the lines of force relative to the chosen $x$ direction. This is true for any pair of orthogonal directions, and therefore really expresses an intrinsic property of the system of curves. (See the diagram in references.)

We use the analytic forms of these properties, which are as follows.

Property I. The equation of the family has the form (3), $y^{\prime \prime \prime}=G y^{\prime \prime}+H y^{\prime \prime 2}$.

Property II. $H=3 /\left[y^{\prime}-\omega(x, y)\right]$.

Property III, IV. Equation (3) is further specialized to

$$
\left(y^{\prime}-\omega\right) y^{\prime \prime \prime}=\left(\lambda y^{\prime 2}+\mu y^{\prime}+\nu\right) y^{\prime \prime}+3 y^{\prime \prime 2},
$$

where $\omega, \lambda, \mu, \nu$ are arbitrary functions of $x$ and $y$.

(6) Property V. $\lambda \omega^{2}+\mu \omega+\nu+\omega_{x}+\omega \omega_{y}=0$.

(7) Property VI. $\quad \lambda_{x}+\left[\left(\nu+\omega_{x}\right) / \omega\right]_{y}=0$.

We impose these conditions successively on the class of all curvature families, defined by (2), thus arriving at each step at a more special subclass for which the properties imposed up to that point could be given as defining theorems. Moreover, the classification is projective, for the properties I-VI are separately projective.

PROPERTy I. All curvature families have this property, since (2) is an equation of type (3).

Property II. $F_{y^{\prime}} / F=3 /\left(y^{\prime}-\omega\right)$. Integrating, we find

$$
F\left(x, y, y^{\prime}\right)=\left[y^{\prime}-\omega(x, y)\right]^{3} u(x, y) .
$$

The force direction $\omega(x, y)$ and the factor $u(x, y)$ are arbitrary. 
Property III, IV. Substituting (8) into (2), we get an equation which already has the form (5) with

(9) $\lambda u=u_{y}, \quad \mu u=u_{x}-3 \omega_{y} u-\omega u_{y}, \quad \nu u=-3 \omega_{x} u-\omega u_{x}$.

Hence curvature families with an $F$ as in (8) have properties I, II, III, IV.

Property V. Condition (6) reduces to

$$
\omega_{x}+\omega \omega_{y}=0 \text {. }
$$

This means that the lines of force $y^{\prime}=\omega(x, y)$ must be a oneparameter family of straight lines.

Property VI. Condition ( 7 ) becomes

$$
\left(\omega_{x} / \omega\right)_{y}=0 \text {. }
$$

From (11) and (10), $\omega_{y y}=0$, so that $\omega$ is linear in $y$. We deduce finally that either $\omega=y / x$ (if we translate the axes) or $\omega=k$ (const.). Hence if curvature trajectories are also dynamical trajectories, the generating family $\left(2^{\prime}\right)$ must be either

or

$$
\text { First case: } \quad y^{\prime \prime}=\left(y^{\prime}-y / x\right)^{3} u(x, y)
$$

$$
\text { Second case: } y^{\prime \prime}=\left(y^{\prime}-k\right)^{3} u(x, y) \text {. }
$$

If we calculate (2) for these special $F$ 's and compare it with (1), we find in the first case that $\psi / \phi=y / x$ (proving that the field is central) and $\phi=u / x^{2}$. The equation of the generating family $\left(2^{\prime}\right)$ may be rewritten more symmetrically

$$
y^{\prime \prime}=\left(x y^{\prime}-y\right)^{3} v(x, y)
$$

with $v$ arbitrary. The components of the corresponding field are $\phi=x v$ and $\psi=y v$. In the second case, $\psi / \phi=k$, so that the field is parallel (center at infinity), and $\phi=u$. If we take the field horizontal, the equation of the generating family $\left(2^{\prime}\right)$ becomes

$$
y^{\prime \prime}=y^{\prime 3} v(x, y)
$$

with $v$ arbitrary. The components of the field are $\phi=v$ and $\psi=0$.

We have thus proved the following theorem.

Theorem. A family of dynamical trajectories due to a positional field of force is resolvable into curvature trajectories when and only when the field is central or parallel. The base doubly-infinite family is then of the form $\left(12_{1}\right)$ or $\left(12_{2}\right)$.

3. Families Derived from Conical Surfaces. In a paper analo- 
gous to the present one we compared dynamical trajectories and sectional families. * A sectional family is defined by taking all the $\infty^{3}$ plane sections of an arbitrary surface and projecting them from some fixed center on to a fixed plane. We found that cones (of any cross-section) were the only surfaces that produced dynamical families. The corresponding fields of force were again central (or parallel), but of a special kind, the force following an inverse square law along any line of force (the constant being allowed to vary for different lines). The Newtonian field was included as a special solution, the cone then being a right circular cone.

We are now able to conclude that these same families (sectional families derived from conical surfaces) are distinguished by being the largest class which are of dynamical, sectional, and curvature types all at once.

4. Comparison of Sectional and Curvature Types. Finally, we might compare the sectional and curvature types with each other. We already know that they have in common the families projected from general cones. To these we can add the family of all circles mentioned above as an example of curvature trajectories, for stereographic projection of the sphere gives the same circles. But with the sphere we have at once all proper quadrics, provided the center of projection is taken on the surface; this follows from the projective character of the work. We can remove the restriction as to the center of projection by another example: orthogonal projection of the plane sections of a sphere gives a family of $\infty^{3}$ ellipses all contained in a circle; $\infty^{2}$ of these are tangent to the circle at one end of their minor axes; these $\infty^{2}$ have the whole family for their curvature trajectories. Thus the sectional families from quadrics and general cones are also curvature trajectories. It can be shown that this is the full answer. $\dagger$

5. Overlapping of the Three Types. We thus have complete knowledge of the possible overlapping of the dynamical, sectional,

* E. Kasner, Dynamical trajectories and the $\infty^{3}$ plane sections of a surface, Proceedings of the National Academy of Sciences, vol. 17 (1931), pp. 370376. Again, sectional families are projectively invariant and they have differential equations of the form (3).

$\dagger$ This result is due to $G$. Comenetz. Another proof has been given by $M$ Halperin. 
and curvature types of triply infinite families of curves. The reresults are summarized in the following table; the two types compared are named in the left-hand column; their intersection is identified in the center; and the number, that is, the infinitude, of (projectively different) common families is given at the right.

Dynamical Sectional: Special central fields or $\quad \infty^{f(1)}$

\section{General cones}

Dynamical Curvature: Any central field

$\infty f(2)$

Sectional Curvature: General cones and

Quadric surfaces

$\infty f(1)+2$

The 2 in the exponent of $\infty$ refers of course to two arbitrary constants, while (according to a notation which I proposed in this Bulletin in 1912, in a review of Riquier's treatise on partial differential equations) $f(1)$ means an arbitrary function of one independent variable, and $f(2)$ an arbitrary function of two independent variables.

Columbia University

\section{ON NEVANLINNA'S WEAK SUMMATION METHOD $\dagger$}

\section{BY A. F. MOURSUND}

1. Introduction. Our principal object in this note is to discuss the function

$$
\begin{array}{r}
\rho_{n}(\beta)=\frac{2}{\pi} \int_{0}^{\pi / 2} \mid \int_{0}^{1} \beta(\log C)^{\beta}(1-t)^{-1}(\log C /(1-t))^{-\beta-1} \\
\quad \times \frac{\sin (2 n t+1) s}{\sin s} d t \mid d s
\end{array}
$$

which, for $\beta>0$ and the "dummy" constant $C \geqq e^{\beta+1}$, plays a role in the theory of summation of Fourier series by Nevanlinna's weak method $\ddagger$ analogous to the role the Lebesgue constants

$\dagger$ Presented to the Society, June 20, 1934.

$\ddagger$ F. Nevanlinna, Über die Summation der Fourier'schen Reihen und Integrale, Översikt av Finska Vetenskaps-Societetens Förhandlingar, vol. 64 (1921-22), A, No. 3, 14 pp. A. F. Moursund, On the Nevanlinna and BosanquetLinfoot summation methods, Annals of Mathematics, (2), to appear. 